\title{
Stereotactic radiosurgery versus whole- brain radiotherapy after intracranial metastasis resection: a systematic review and meta-analysis
}

Nayan Lamba ${ }^{1,2 \dagger}$, Ivo S. Muskens ${ }^{1,3+}$, Aislyn C. DiRisio ${ }^{1}$, Louise Meijer ${ }^{3}$, Vanessa Briceno ${ }^{4}$, Heba Edrees ${ }^{4}$, Bilal Aslam ${ }^{4}$, Sadia Minhas, , Joost J. C. Verhoeff5, Catharina E. Kleynen ${ }^{5}$, Timothy R. Smith', Rania A. Mekary ${ }^{1,4}$ and Marike L. Broekman ${ }^{1,3^{*}}$

\begin{abstract}
Background: In patients with one to three brain metastases who undergo resection, options for post-operative treatments include whole-brain radiotherapy (WBRT) or stereotactic radiosurgery (SRS) of the resection cavity. In this meta-analysis, we sought to compare the efficacy of each post-operative radiation modality with respect to tumor recurrence and survival.

Methods: Pubmed, Embase and Cochrane databases were searched through June 2016 for cohort studies reporting outcomes of SRS or WBRT after metastasis resection. Pooled effect estimates were calculated using fixed-effect and random-effect models for local recurrence, distant recurrence, and overall survival.

Results: Eight retrospective cohort studies with 646 patients (238 with SRS versus 408 with WBRT) were included in the analysis. Comparing SRS to WBRT, the overall crude risk ratio using the fixed-effect model was 0.59 for local recurrence (95\%-Cl: 0.32-1.09, I': 3.35\%, P-heterogeneity $=0.36,3$ studies), 1.09 for distant recurrence (95\%-Cl: 0.74-1.60, I': 50.5\%, P-heterogeneity $=0.13 ; 3$ studies), and 2.99 for leptomeningeal disease $\left(95 \% \mathrm{Cl} 1.55-5.76 ; I^{2}: 14.4 \%\right.$ p-heterogeneity: 0. $28 ; 2$ studies). For the same comparison, the risk ratio for median overall survival was 0.47 (95\% Cl: $0.41-0.54 ; 1^{2}: 79.1 \%$, P-heterogeneity $<0.01 ; 4$ studies) in a fixed-effect model, but was no longer significant $(0.63 ; 95 \%-\mathrm{Cl}$ : 0.40-1.00) in a random-effect model. SRS was associated with a lower risk of leukoencephalopathy (RR: 0.15, 95\% Cl: 0.07-0.33, 1 study), yet with a higher risk of radiation-necrosis (RR: 19.4, 95\% Cl: 1.21-310, 1 study).
\end{abstract}

Conclusion: Based on retrospective cohort studies, the results of this study suggest that SRS of the resection cavity may offer comparable survival and similar local and distant control as adjuvant WBRT, yet may be associated with a higher risk for developing leptomeningeal disease. Future research on SRS should focus on achieving a better understanding of the various factors that may favor SRS over WBRT.

Keywords: Brain Metastasis, Resection, Whole brain radiation, Stereotactic radiosurgery, Meta-analysis

\footnotetext{
* Correspondence: m.l.d.broekman-4@umcutrecht.nl

${ }^{\dagger}$ Equal contributors

'Cushing Neurosurgery Outcomes Center (CNOC), Department of Neurosurgery, Brigham and Women's Hospital, Harvard Medical School, Boston, MA, USA

${ }^{3}$ Department of Neurosurgery, Brain Center Rudolf Magnus, University Medical Center Utrecht, HP G03.124, PO Box 855003508 GA Utrecht, The Netherlands

Full list of author information is available at the end of the article
} 


\section{Background}

Brain metastases are an increasingly common complication of systemic cancers and represent a significant source of morbidity and mortality in cancer patients [1-5]. Approximately, $20-40 \%$ of cancer patients with primary extracranial cancer will develop brain metastases during the course of their disease [6]. In the United States alone, this represents about 98,000 to 170,000 new diagnoses each year $[6,7]$. Median survival without treatment is estimated at 1 month, and increases to 3-12 months when cranial radiation therapy is used [8].

Traditionally, the standard of care for patients with solitary brain metastasis has been resection plus whole-brain radiotherapy (WBRT) [4, 7]. Resection allows for histopathological examination of the tissue and has been shown to improve neurological symptoms, functional independence, and survival [5]. Moreover, several studies found that when resection was followed by adjuvant WBRT, it resulted in improved intracranial tumor control and lower rates of neurologic deaths compared to resection alone [9-13]. One randomized controlled trial (RCT) assigned patients to WBRT or observation following either initial surgery or SRS. While the researchers did not find the addition of WBRT to affect overall survival when compared to observation, they did find that following either surgery or SRS, the addition of WBRT reduced the 2-year relapse rate when compared to surgery without adjuvant WBRT. More specifically, WBRT reduced the probability of relapse from 59 to $27 \%$ following surgery. A similar trend was noted following SRS, in which adjuvant WBRT reduced the probability of relapse from 31 to $19 \%$. While the former result is consistent with what other experiments have also demonstrated (i.e. improved disease control with adjuvant WBRT following resection), the latter result is novel in that it suggests a possible role for adjuvant SRS in the management of brain metastases [13]. The fact that SRS followed by WBRT offered better control than SRS alone, along with the fact that surgery plus WBRT led to improved outcomes as compared to surgery alone, suggests the possibility that SRS following surgery may offer similarly improved rates of disease control without the sequelae associated with radiation exposure to the entire brain.

Furthermore, WBRT is associated with both short and long-term neurological complications, such as radiationinduced edema, leukoencephalopathy, and cognitive deficits $[2,7,10]$. Given that patients with systemic cancer are now living longer due to improved extracranial anti-tumor strategies, concerns related to these delayed, radiation-induced neurotoxic effects are prompting neuro-oncologists to assess alternatives to WBRT $[4,14]$. Many centers are therefore using targeted techniques with limited radiation exposure, such as stereotactic radiosurgery (SRS). SRS is associated with fewer global cognitive side effects, CNS preservation, and better quality-of-life [2, 4, 7, 15].
In this study, we used the current published literature to compare local recurrence, distant recurrence, leptomeningeal disease, and overall patient survival amongst patients who underwent resection plus SRS to patients who underwent resection plus WBRT for treatment of one to three intracranial metastases. Given the available data and abovementioned RCT, we hypothesize that SRS may lead to similar rates of local control as compared to WBRT following resection, but, based upon the targeted scope of SRS, may lead to poorer rates of distant control and overall patient survival.

\section{Methods}

This meta-analysis was done in accordance with the Preferred Reporting Items for Systematic Reviews and MetaAnalyses (PRISMA) statement [16]. PubMed, EMBASE, and Cochrane databases were searched on 6-25-2016 for studies comparing outcomes of intracranial metastasis resection followed by WBRT versus intracranial metastasis resection followed by SRS using the following keywords: Whole-Brain Radiotherapy, Stereotactic Radiosurgery, resection and intracranial metastasis with synonyms (Appendix 1). Language was limited to English.

\section{Eligibility criteria and study selection}

Inclusion criteria consisted of any study that described adult patients ( $\geq 18$ years of age) with a diagnosis of systemic cancer who underwent neurosurgery followed by whole-brain radiotherapy (WBRT) or by stereotactic radiosurgery (SRS) for treatment of up to three metastases to the brain. SRS was defined as a single or few fractions of high dose radiation to a small intracranial target that was encompassed by at least $50 \%$ of the prescribed dose [17]. Because Intraoperative Radiation Therapy (IORT) meets most of the salient features of SRS as described by the Radiation Oncology Therapy Group protocol, $[18,19]$ studies reporting IORT were included within the SRS treatment groups. Studies reporting on the use of Local Brain Radiotherapy (LBRT), however, were excluded for not meeting the criteria of SRS. Studies reporting outcomes on patients receiving prior WBRT and studies that did not compare post-operative WBRT to post-operative SRS were also excluded. After title and abstract screening, remaining articles were read full-text. Three authors (NL, IM, and LM) performed full-text screening and two (NL and LM) extracted the data. One senior author (MB) reviewed the included articles and the extracted data. Disagreements were solved by discussion.

\section{Data extraction and management}

The following data were extracted from each study whenever possible: author and year of published articles, number of patients in the study, patient characteristics (inclusion criteria, primary tumor type, age, type of 
intervention (whole-brain radiotherapy, stereotactic radiosurgery, radiation dose and regimen, time between resection and radiation), number of intracranial lesions at presentation, extent of extracranial disease), duration of follow-up, and primary and secondary outcomes of the study. When available, relative risks (RR) comparing SRS to WBRT were extracted for local recurrence risk, distant recurrence, leptomeningeal disease, and overall survival. Duration of overall survival (OS) and progression free survival (PFS) for both local and distant recurrence was also noted. Furthermore, data on the number of patients that died from neurological causes and on observed neurotoxicity were extracted. In one study reporting outcomes of multiple metastases per patient, the reported percent recurrence by number of cavities was considered as equivalent to the percent recurrence by number of patients, as the number of metastases per patient approximated 1:1 [20].

Study quality was assessed by the New-Castle Ottawa Scale, and the quality of our recommendation was assessed using the Grading of Recommendations, Assessment, Development and Evaluations (GRADE) criteria. Both assessments were made by two authors (NL and IM) independently [21, 22]. Discrepancies were solved by discussion.

\section{Data analysis}

To minimize heterogeneity amongst studies being compared, only cohort studies were included in the metaanalysis. Data analysis was performed using Comprehensive Meta-Analysis (CMA) Version 3 (Biostat, Inc., Englewood, NJ, USA). Overall fixed and random risk ratios were calculated for the same outcomes, depending on the available data in the articles. I-squared values were calculated to assess heterogeneity. For median overall survival, relative risks were calculated based on group size and median overall survival both for SRS and WBRT [23]. Meta-regression analysis on continent, age, and journal impact-factor was used when possible to assess sources of heterogeneity.

\section{Results}

The search strategy resulted in 9908 articles after removal of duplicates. After screening for titles and abstracts, 410 full-texts were screened. Eight retrospective cohort studies provided outcomes for 646 patients treated with WBRT $(\mathrm{n}=408)$ or SRS $(\mathrm{n}=238)$ following resection (Fig. 1) [5, 18, 20, 24-28]. The most common primary tumor histologies were non-small cell lung cancer and colon cancer. Mean group size was 30 patients for SRS and 51 for WBRT. The mean age for the SRStreated groups was 58 while the mean age for the WBRT-treated groups was 56 years. Mean radiation dose was single dose 18 Gy for SRS and fractionated dose
35 Gy for WBRT. The Newcastle-Ottawa Scale (NOS) varied between 6 and 8 among the studies (Table 1) and all studies were deemed of very low quality based on GRADE criteria (Table 2).

\section{Local recurrence}

Data regarding local recurrence was available in five out of the eight studies included in our review [5, 18, 20, 26, 27]. The local recurrence incidence ranged from 0 to $60 \%$ in patients treated with adjuvant SRS and from 11 to $24 \%$ in patients treated with adjuvant WBRT. While no study reported a statistically significant difference in local recurrence rate between the two treatment groups, four of the five studies that included data on local recurrence did note differences in these rates. One study reported a near-significant hazard ratio $(p=0.09)$ that disfavored SRS over WBRT by a $68 \%$ higher rate of distant recurrence [18]. One Italian study from 1996 that did not specifically report a $p$-value comparing just these two treatment arms did find that 5 patients treated with adjuvant SRS were nearly four times as likely to experience local recurrence as compared to 14 patients treated with adjuvant WBRT [27]. Two other studies favored SRS for achieving local control, with percent differences in local recurrence ranging from 3 to $17 \%$ in favor of SRS $[20,26]$. Only the study by Lee et al. demonstrated near-equal rates of local recurrence for both treatment groups, with a local recurrence of 11 and 12\% for WBRT and SRS treatment groups, respectively [5].

The pooled risk ratio comparing local recurrence incidence between SRS vs. WBRT groups was 0.79 (95\% CI: 0.48-1.29; fixed effects model) and demonstrated no significant difference in rates of local recurrence (Fig. 2). Results from the random effects model were similar (RR: $0.88 ; 95 \%-\mathrm{CI}=0.40-1.92$ ) and moderate heterogeneity was observed $\left(\mathrm{I}^{2}=49.44 \%, \quad \mathrm{p}\right.$-heterogeneity $=0.10, \quad 3$ studies). This analysis was based on 163 patients receiving post-operative WBRT and 138 patients receiving postoperative SRS.

Univariate meta-regression on age $(p=0.02)$ and continent $(p=0.04)$ were identified as a source of heterogeneity. With increasing age, the RR for local recurrence was in favor of SRS as compared to WBRT. Furthermore, Europe, in comparison to North America was associated with a RR for local recurrence in favor of WBRT. Journal impact factor was not identified as a source of heterogeneity $(p=0.97)$.

\section{Distant progression}

Data regarding distant progression was available in three out of the eight studies included in our study (Fig. 3) [5, $20,26]$. The distant progression incidence ranged from 6 to $50 \%$ in patients treated with adjuvant SRS and from 17 to $44 \%$ in patients treated with adjuvant WBRT $[5,20,26]$. 
Articles found in Pubmed ( $\mathrm{n}=5066)$

Articles found in Embase ( $\mathrm{n}=4659)$

Articles found in Cochrane Library $(n=423$ )

Total Articles $(\mathrm{n}=10,148)$

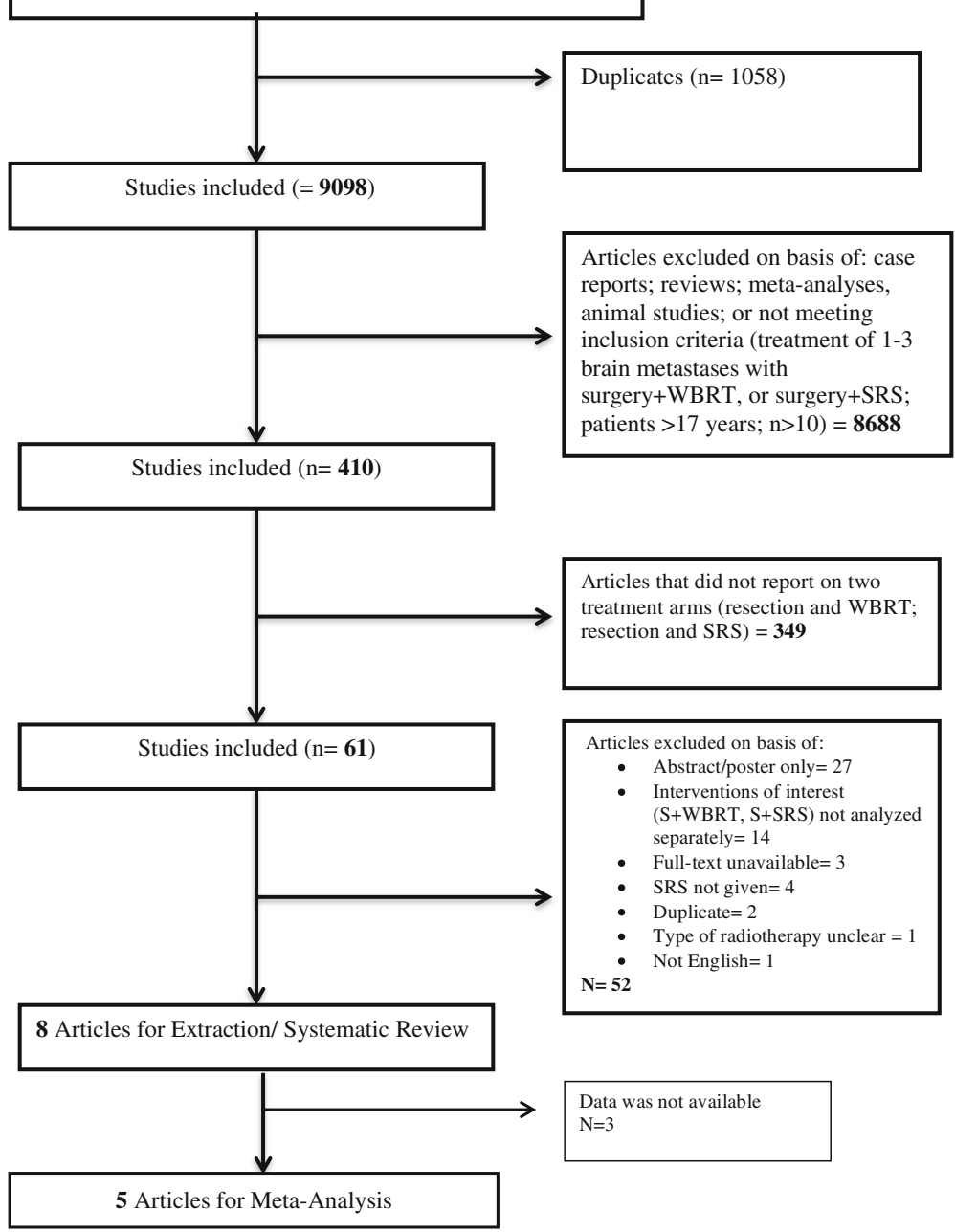

Fig. 1 Study selection process of the included articles

Three of the studies, while not reaching statistical significance, also reported distant progression rates that were 6 to $11 \%$ higher in SRS patients as compared to WBRT patients $[20,26]$. One study reported better distant control in $17 \mathrm{pa}-$ tients treated with SRS as compared to 109 treated with WBRT [5]. One additional study reported a statistically significant distant progression hazard ratio of 2.17 for patients treated with SRS (single dose range: 14 to $24 \mathrm{~Gy}$ ) after resection compared to those treated with WBRT (dose: 30 Gy in 10 fractions or 37.5 Gy in 15 fractions) [18]. The pooled risk ratio comparing distant recurrence rates between SRS versus WBRT was 1.09 (95\%-CI: 0.74-1.60; fixed effects model) and demonstrated no significant difference in rates of distant recurrence (Fig. 3). Results from the random effects model were similar (RR: 0.94; 95\%-CI: $0.39-2.26)$, and moderate heterogeneity was observed $\left(\mathrm{I}^{2}=\right.$
$50.5 \%$, p-heterogeneity $=0.28,3$ studies, Fig. 3). Continent $(p=0.52)$, age $(p=0.12)$, and journal impact factor $(p=$ $0.52)$ were not identified as sources of heterogeneity. This analysis was based on 163 patients receiving postoperative WBRT and 138 patients receiving postoperative SRS.

\section{Leptomeningeal disease}

Two studies reported on the development of leptomeningeal disease (LMD) following radiotherapy [18, 20]. Retrospectively, SRS treatment relative to WBRT was associated with a higher risk of LMD occurrence as per Hsieh et al. [18] (HR:2.44; 95\%-CI:1.15-5.18) and Patel et al. [24] (HR:5.67; 95\%-CI: 1.50-21.51).

The pooled relative risk for the development of LMD following postoperative SRS to postoperative WBRT was 2.99 (95\% CI 1.55-5.76), indicating that resection 


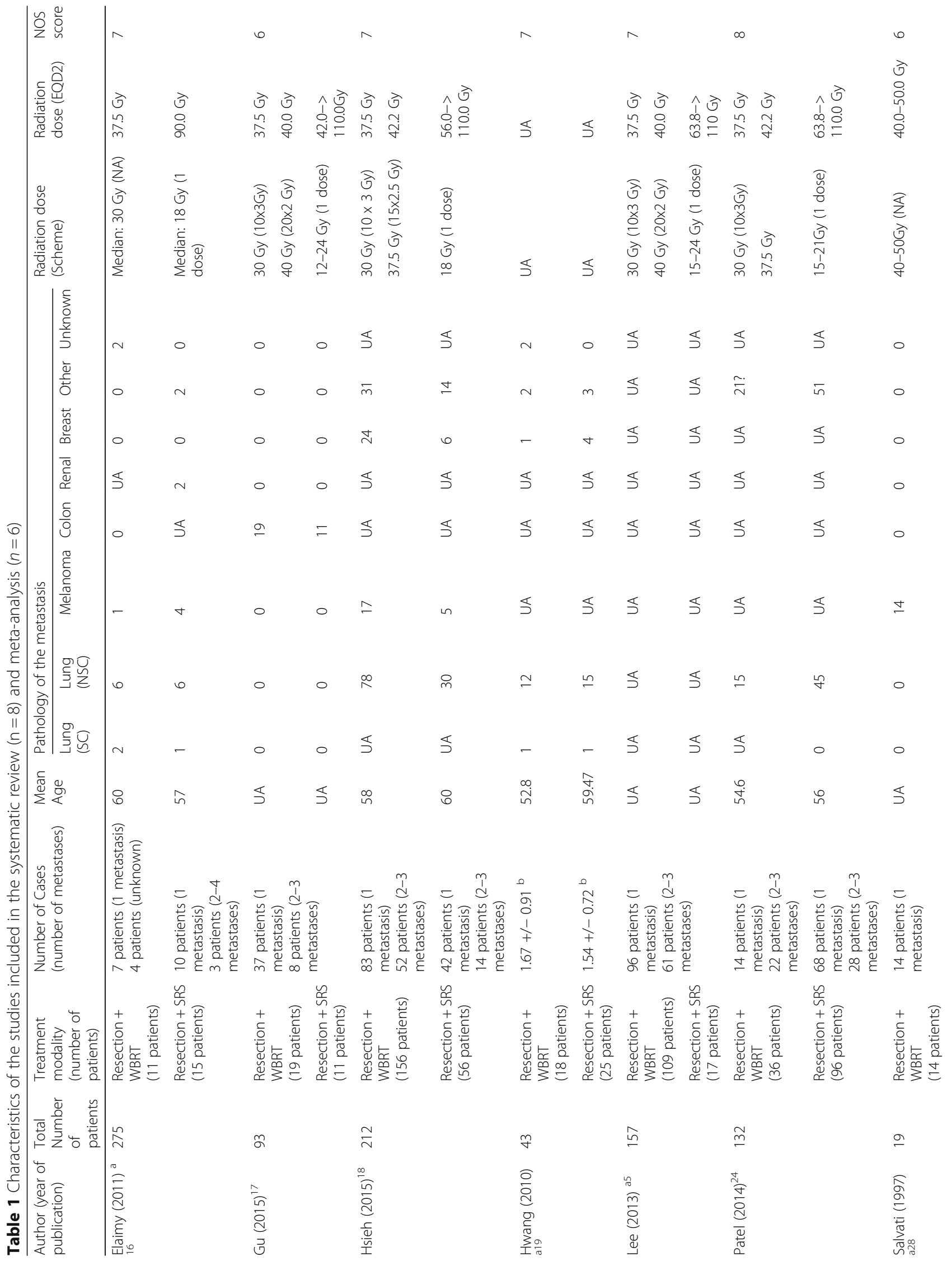


Lambs et al. Radiation Oncology (2017) 12:106

Page 6 of 12

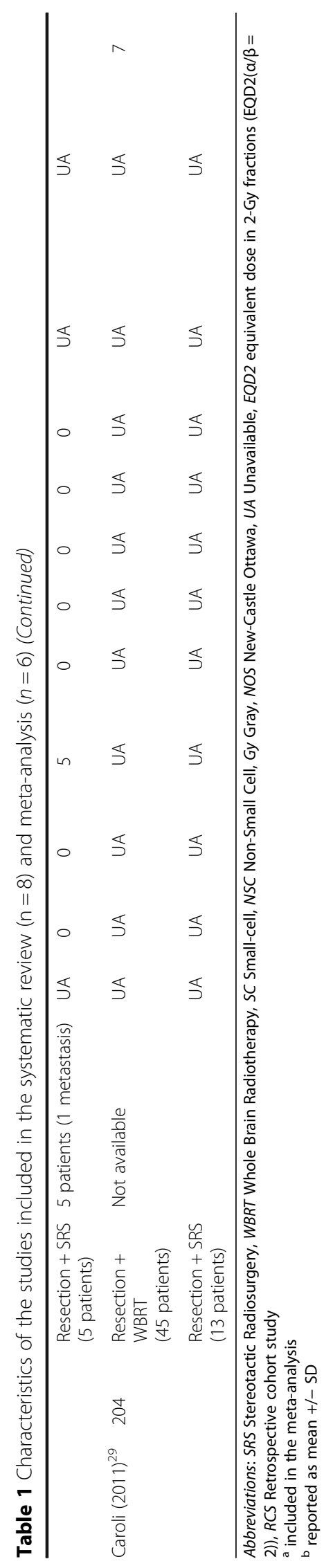


Table 2 The Grading of Recommendations, Assessment, Development and Evaluations (GRADE) Criteria was used to assess the level of evidence for each outcome

\begin{tabular}{llllllll}
\hline Outcomes & Type of Evidence & Quality & Consistency & Directness & Effect Size & Total & Overall Quality \\
\hline Local Control & +2 & -2 & 0 & 0 & 0 & 0 & Very low \\
Distant Progression & +2 & -2 & 0 & 0 & 0 & 0 & Very low \\
LMD & +2 & -2 & -1 & 0 & +1 & 0 & Very low \\
Overall Survival & +2 & -2 & -1 & 0 & 0 & -1 \\
\hline
\end{tabular}

Type of evidence is based on the study design of the included studies and ranges from +2 to +4 . Study quality is graded based on blinding and allocation, follow up and withdrawals, sparsity of data, or methodological concerns and ranges from -3 to 0 . Consistency is graded based on heterogeneity of populations and study end points with respect to one another and included populations and ranges from -1 to +1 . Directness is graded based on generalizability of the included results and is graded from -2 to 0 . Effect size is graded on the value of the RR or OR and is graded from 0 to +2 . The overall quality of the recommendations that can be made based on the included studies includes the following categories: high (at least 4 points overall), moderate ( 3 points), low ( 2 points), and very low ( 1 point or less)

followed by SRS increases the risk for developing LMD as compared to resection followed by WBRT (Fig. 4). There was low heterogeneity $\left(\mathrm{I}^{2}: 14.4 \%\right.$ p-heterogeneity: $0.28,2$ studies) between the included studies. This remained significant in the random effects model (RR: 3.09, 95\% CI: 1.47-6.48). The analysis is based on 192 patients receiving resection followed by WBRT and 152 patients receiving resection followed by SRS.

\section{Overall survival}

Only one study out of the 8 included in this review found a significant difference in survival between patients treated with SRS following resection as opposed to WBRT [24]. In their 2011 institutional study, Elaimy et al. retrospectively compared six different treatment regimens in patients with metastatic lesions in the brain [24]. Using SRS only as a reference, they found that 15 selected patients treated with SRS following resection had a $68 \%$ lower risk of death as compared to 65 patients treated with SRS alone $(p=0.02)$. In contrast, they reported a slightly higher risk of death $(\mathrm{HR}=1.04)$ in 11 patients treated with WBRT after resection as compared to SRS alone [24]. While the other eight

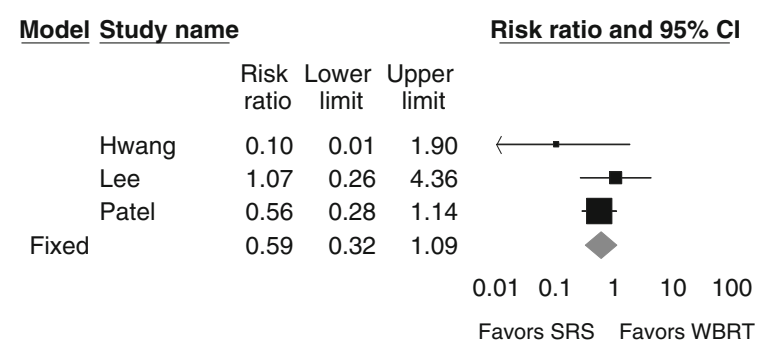

I-squared= 3.35\%; P-heterogeneity= 0.36

Fig. 2 Local recurrence after SRS versus WBRT following neurosurgical resection. Forest plot represents the risk ratio for local recurrence of intracranial metastasis following resection and SRS versus resection and WBRT (95\% confidence interval [CI]) with 5 cohort studies in adults ( $n=138$ patients (SRS) and 163 patients (WBRT)). Solid squares represent the point estimate of each study and the diamond represents the pooled estimate of the risk ratio. The $P$ and $P$ values for heterogeneity are shown studies did not report significant differences in survival between the two treatment groups, two studies were slightly in favor of SRS, $[18,26]$ while three $[5,27,28]$ indicated slightly better outcomes after WBRT, with one even reaching a near-significant level in favor of WBRT $(p=0.06)[5]$.

The pooled relative risk for median overall survival comparing patients treated with SRS after resection to those treated with WBRT was 0.51 (95\%-CI: 0.44-0.54; I²: 86.6\%, p-heterogeneity $<0.01 ; 4$ studies), suggesting a favorable outcome for WBRT with considerable heterogeneity (Fig. 5) [5, 24, 26, 27]. However, the overall survival was non-significant using the random-effect model (RR: 0.78; 95\%-CI: 0.40-1.00) [5, 24, 26, 27]. The analysis was based on 174 patients receiving postoperative WBRT and 153 patients receiving postoperative SRS. Univariate meta-regression on continent $(p<0.01)$ and journal impact factor $(p<0.01)$ were identified as sources of heterogeneity. Europe and Asia, in comparison to North America, were associated with a RR for overall survival in favor of SRS. A lower impact factor journal showed a RR in favor of

I-squared= 50.5\%; P-heterogeneity $=0.13$

Fig. 3 Distant progression after SRS versus WBRT following neurosurgical resection. Forest plot represents the risk ratio for distant recurrence of intracranial metastasis following resection and SRS versus resection and WBRT (95\% confidence interval [CI]) with 4 cohort studies in adults ( $n=138$ patients (SRS) and 163 patients (WBRT)). Solid squares represent the point estimate of each study and the diamond represents the pooled estimate of the risk ratio. The $P^{2}$ and $P$ values for heterogeneity are shown 


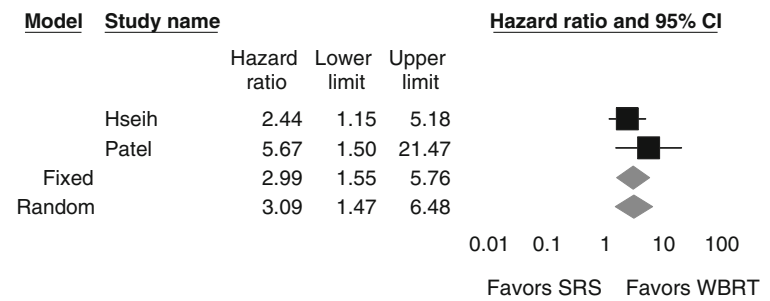

I-squared= 14.4\%; P-heterogeneity $=0.28$

Fig. 4 Leptomeningeal Disease after SRS versus WBRT following neurosurgical resection. Forest plot represents the risk ratio for overall survival of intracranial metastasis following resection and SRS versus resection and WBRT (95\% confidence interval [CI]) with 2 cohort studies in adults ( $n=152$ patients (SRS) and 192 patients (WBRT)). Solid squares represent the point estimate of each study and the diamond represents the pooled estimate of the risk ratio. The $P^{2}$ and $P$ values for heterogeneity are shown

WBRT as compared to SRS. Age was not identified as a source of heterogeneity $(p=0.14)$.

\section{Radiation necrosis and leukoencephalopathy}

Radiation necrosis and leukoencephalopathy were each reported in one study. Patel et al. found that symptomatic radiation necrosis, requiring the use of steroid or other interventions, was $27 \%$ in the SRS group and $0 \%$ in the WBRT group $(p<0.01)$. After 1 year, the rate of radiographic leukoencephalopathy was $7 \%$ following SRS vs. $47 \%$ following WBRT $(p<0.01)$. The relative risk of leukoencephalopathy for SRS compared to WBRT in this study was 0.15 (95\% CI: 0.11-0.21).

\section{Discussion}

The present study demonstrated that there was no statistically significant difference in local recurrence or

\begin{tabular}{|c|c|c|c|c|c|c|c|}
\hline \multirow[t]{2}{*}{ Model } & \multirow[t]{2}{*}{ Study name } & & & & \multicolumn{3}{|c|}{ Risk ratio and $95 \% \mathrm{CI}$} \\
\hline & & $\begin{array}{l}\text { Risk } \\
\text { ratio }\end{array}$ & $\begin{array}{l}\text { Lower } \\
\text { limit }\end{array}$ & $\begin{array}{l}\text { Upper } \\
\text { limit }\end{array}$ & & & \\
\hline & Elaimy & 0.42 & 0.31 & 0.55 & & & \\
\hline & Hwang & 0.45 & 0.39 & 0.53 & & & \\
\hline & Lee & 1.88 & 0.79 & 4.47 & & & \\
\hline & Salvati & 1.71 & 0.46 & 6.46 & & & \\
\hline Fixed & & 0.47 & 0.41 & 0.54 & & 1 & \\
\hline \multirow[t]{2}{*}{ Random } & & 0.63 & 0.40 & 1.00 & & $>$ & \\
\hline & & & & & $0.01 \quad 0.1$ & 10 & 100 \\
\hline
\end{tabular}

I-squared= 79.1\%; P-heterogeneity $<0.01$

Fig. 5 Survival after SRS versus WBRT following neurosurgical resection. Forest plot represents the risk ratio for overall survival of intracranial metastasis following resection and SRS versus resection and WBRT (95\% confidence interval [Cl]) with 4 cohort studies in adults ( $n=153$ patients (SRS) and 174 patients (WBRT)). Solid squares represent the point estimate of each study and the diamond represents the pooled estimate of the risk ratio. The $P^{2}$ and $P$ values for heterogeneity are shown distant recurrence between patients receiving SRS versus WBRT following neurosurgical resection of brain metastases in retrospective cohorts. Of note, there was a significant increased risk for the development of LMD in patients who received postoperative SRS as compared to WBRT, and a possible survival benefit to WBRT shown only in the fixed effects model with considerable heterogeneity. Though there was little published on leukoencephalopathy and radiation necrosis in these patients, it appeared that SRS reduced the risk of leukoencephalopathy and increased the risk for radiation necrosis as compared to WBRT. These results were in support of our hypothesis regarding similar rates of local control between the two treatment arms, but against our hypothesis predicting that SRS would lead to poorer rates of distant control. The possible survival benefit with WBRT in the fixed effects model was consistent with our hypothesis.

No randomized controlled trials have yet been completed for post-operative radiation modalities. However, a meta-analysis on the effect of the addition of WBRT to SRS or surgery did show less intracranial disease progression, but no effect on overall survival [29]. This is in line with the results of a trial evaluating additional WBRT to SRS that found improved intracranial tumor control but no effect on survival [15]. However, the addition of SRS to WBRT was found to result in longer survival in a randomized trial [30]. A systematic review evaluating linac-based SRS to resection cavities concluded that this treatment strategy provided good local control rates but poor distant intracranial control. This is different form our study that focused on a comparison between SRS and WBRT after resection [31].

Other studies have reported on the high risk of LMD in patients receiving SRS to the resection cavity, and have cited incidence rates around 12-14\% [32-34]. Breast cancer metastases have been shown to have a particularly high rate of LMD [32-34]. Additionally, many studies reported on the adverse side effects of WBRT, including physical symptoms such as alopecia, somnolence, hearing loss, skin changes, and neurocognitive decline, manifested as memory loss and learning impairment $[2,7,18,26]$. In particular, the cognitive decline following WBRT has been linked to leukoencephalopathy, which is supported by the increased risk for leukoencephalopathy in patients receiving post-operative WBRT [20, 35] Another study, evaluating 59 patients treated with primary SRS, found that even after multiple courses of SRS, quality of life (QOL), measured with the EQ-5D instrument, was preserved in 77\% of patients at 12 month follow-up [36]. Thus, considering that our present analysis demonstrates similar results with respect to local recurrence, distant progression, and potentially survival in post-operative SRS patients, SRS is a valid treatment option that can help maintain neurocognition and QOL. 
In patients with a limited number of brain metastases receiving resection, the results of this study suggest that it is important to understand the risks and benefits of both WBRT and SRS. The possibility for an improved quality of life should be considered when discussing treatment options. Although there is still not a clear formula to guide use of SRS or WBRT following resection, the fact that SRS may offer similar tumor control as WBRT, with the potential for fewer cognitive side effects and less invasiveness, suggests that SRS should be considered as a valid treatment option for brain metastases [26, 37]. While it has been widely acknowledged that WBRT is associated with cognitive decline, SRS has also been associated with radiation induced necrosis [38]. While these studies showed a potential survival improvement in WBRT as compared to SRS in the random effects model, it is important to note that the fixed effects model was not significant and that the considerable heterogeneity between the studies limits the conclusions that can be drawn from this. Both continent and journal impact factor were identified as significant sources of heterogeneity. Lastly, we demonstrated an increased risk for LMD in patients receiving postoperative SRS to the resection cavity, which is an important outcome to discuss with patients considering SRS.

\section{Limitations and strengths}

A major limitation of this review and meta-analysis was the heterogeneity among the included studies. Amongst the nine studies included, one included only colon cancer patients, one included melanoma patients only, and the remaining six consisted of patient populations with varying proportions of melanoma, colon, renal, breast, and unknown histologies $[5,18,20,24,26,28]$. Additionally, the number of brain metastases was not reported homogenously between studies, and reported outcomes were not specific to the number of brain metastases and treatment modalities. Therefore, it was not possible to perform a subgroup analysis by histology type or number of metastases, or address potential sources of heterogeneity by these covariates. This is most likely of importance, because certain tumors, such as melanoma, colon, and renal carcinomas, are considered radioresistant for WBRT $[10,39,40]$ and it is possible that outcomes could differ in patients with only a single metastasis $[41,42]$. Of note, one study included in the analysis reported patients with 2-4 metastases in a single group, but noted that the number of metastases did not have an effect on the outcomes [24]. A further limitation of this analysis was that some studies reported death as a hazard ratio, while other used the Kaplan Meier method. Reporting a hazard ratio is a more accurate method and the variation in reporting limits the strength of the conclusions. Lastly, the studies included in the analysis were all retrospective in nature. Therefore, the different treatmentstrategies implemented for each set of patients were the result of the tumor boards' selection of patients whom they felt would benefit from that regimen, resulting in a selection bias. This is further complicated by the different forms of radiation administered in each study, as some studies described outcomes of IORT instead of SRS [18]. No studies were identified that compared WBRT with hypofractionated SRS, which has become a viable treatment strategy for lesions not treatable with a single fraction SRS [43, 44].

Despite these limitations, this meta-analysis based on cohort studies evaluated several different outcomes after extensive review of the literature. To our knowledge, this is the first meta-analysis on this topic. The use of both random- and fixed-effect models together with heterogeneity analysis resulted in a critical evaluation of our outcomes. Furthermore, an increased risk for the development of LMD after SRS compared to WBRT following surgical resection was identified.

\section{Future directions}

There is a clear necessity for the improvement of care for brain metastasis patients as they tend to live longer. Currently, several treatment strategies exist for brain metastasis, but data on the most effective strategy is lacking. Factors such as radiation dose, treatment timing, chemotherapy combinations among others should be evaluated in future studies in order to evaluate the most effective treatment modalities. The wide range of treatment modalities also provides a potential to strive towards more individualized patient care. In addition, evaluated outcomes should not be limited to survival or progression and should include quality of life. Indeed, currently a phase III randomized trial is being conducted to evaluate postoperative SRS versus WBRT in brain metastasis patients (NCT01372774) [45]. Primary endpoints will be evaluation of survival and neurocognitive progression and secondary endpoints will be QOL, adverse events, and functional independence among others. Last, the use of "big data" may provide a method to evaluate these outcomes more thoroughly.

\section{Conclusions}

This meta-analysis suggested that post-operative, localized SRS may offer local protection of a similar degree as postoperative WBRT in patients with one to three lesions. WBRT as compared to SRS, however, seemed to offer better protection from LMD and may offer better protection from distant progression of intracranial metastases with improved survival, albeit not reaching statistical significance in this meta-analysis. However, because WBRT has been consistently associated with post-operative development of physical and cognitive symptoms, SRS is a valid alternative to consider, and can be used in multiple sessions if distant metastases eventually do develop. Notably, the long-term risks of radiation from SRS must also be considered and weighed against the long-term risks of WBRT. 


\section{Appendix 1}

Search String. (Search performed on 6-25-16)

PUBMED (5066): (Surg*[Tw] OR resect*[Tw] OR operation*[Tw] OR operativ*[Tw] OR "Surgical Procedures, Operative"[MeSH Terms] OR "Neurosurgical Procedures"[Mesh:NoExp] OR "Neurosurgery"[Mesh] OR "Craniotomy"[Mesh] OR "Neurosurgery"[Mesh] OR "Brain/surgery"[Mesh] OR craniotom*[tw] OR neurosurg*[tw]) AND

(Radiotherap* [Tw] OR Radiosurg* $[T w]$ OR LINAC[Tw] OR linear accelerator[Tw] OR (Gamma Knife[Tw]) OR Cyberknife[Tw] OR "x knife"[Tw] OR stereotactic*[Tw] OR fraction*[Tw] OR irradiat*[Tw] OR radiat* OR WBRT[Tw] OR SRS[Tw] OR SFRT[Tw] OR Radiotherapy[MeSH Terms] OR Dose fractionation[MeSH Terms] OR Cranial Irradiation[MeSH Terms] OR Radiosurgery[MeSH Terms]) AND ((Brain[Tw] OR "Brain"[MeSH Terms] OR Cerebr*[Tw] OR "Cerebrum"[MeSH Terms] OR Cerebell*[Tw] OR Intracranial[Tw]) AND (Metasta*[Tw] OR Neoplasm Metastasis[MeSH Terms])) Filters: English

Embase (4659): (surg* OR resect* OR operation* OR operativ* OR craniotom* OR neurosurg* OR 'neurosurgery'/exp OR 'neurosurgery' OR 'brain surgery'/exp OR 'brain surgery' OR 'craniotomy'/exp OR 'craniotomy') AND (radiotherp* OR radiosurg* OR linac OR 'gamma knife'/exp OR 'gamma knife' OR 'cyberknife'/exp OR cyberknife OR 'x knife' OR stereotactic OR irradiati* OR radiat* OR wbrt OR srs OR SFRT OR 'radiotherapy'/exp OR 'radiotherapy' OR 'gamma knife radiosurgery'/ exp OR 'gamma knife radiosurgery' OR 'brain radiation'/exp OR 'brain radiation' OR fraction* OR 'radiation dose'/exp OR 'linear accelerator'/exp OR 'linear accelerator' OR 'stereotactic radiosurgery'/exp OR 'stereotactic radiosurgery' OR 'stereotactic body radiation therapy'/exp OR 'stereotactic body radiation therapy') AND ((brain OR cerebr* OR cerebell* OR intracranial OR 'cerebellum'/exp OR 'cerebellum' OR 'brain'/ exp OR 'brain' OR 'brain tumor'/exp OR 'brain tumor') AND (metastas* OR 'brain metastasis'/exp OR 'brain metastasis')) ([english]/lim) AND [embase]/lim NOT [medline]/lim

Cochrane (432):

MeSH descriptor: [Neurosurgery] explode all trees

\#2

MeSH descriptor: [Surgical Procedures, Operative] explode all trees

\#3

MeSH descriptor: [Neurosurgical Procedures] this term only

\#4

MeSH descriptor: [Craniotomy] explode all trees

\#5

Surg* or resect* or operation* or operativ* or craniotom* or neurosurg \#6

\#1 or \#2 or \#3 or \#4 or \#5

\#7

MeSH descriptor: [Radiotherapy] explode all trees

\#8

MeSH descriptor: [Dose Fractionation] explode all trees

\#9

MeSH descriptor: [Cranial Irradiation] explode all trees

$\# 10$

MeSH descriptor: [Radiosurgery] explode all trees

\#11

Radiotherap* or Radiosurg* or LINAC or "linear accelerator" or "Gamma Knife" or Cyberknife or "x knife" or stereotactic* or fraction* or irradiat* or radiat* or WBRT or SRS or SFRT

$\# 12$

$\# 7$ or \#8 or \#9 or \#10 or \#11

\#13

Brain or Cerebr* or Cerebell* or Intracranial

\#14

MeSH descriptor: [Cerebrum] explode all trees

\#15

MeSH descriptor: [Brain] explode all trees

\section{(Continued)}

\#16

$\# 13$ or \#14 or \#15

\#17

metasta* $^{*}$

\#18

MeSH descriptor: [Neoplasm Metastasis] explode all trees

\#19

$\# 17$ or \#18

\#20

$\# 19$ and \#16

\#21

\#6 and \#12 and \#20

\section{Abbreviations}

CMA: Comprehensive Meta-Analysis; EQD2: Equivalent dose in 2-Gy fraction; GRADE: Grading of Recommendations, Assessment, Development and Evaluations; Gy: Gray; HR: Hazard ratio; IORT: Intraoperative radiotherapy; LMD: Leptomeningeal disease; MMSE: Mini-Mental State Examination; NOS: Newcastle-Ottawa Scale; NSC: Non-Small Cell; OS: Overall survival; PFS: Progression free survival; PRISMA: Preferred Reporting Items for Systematic Reviews and Meta-Analyses; QOL: Quality of Life; RCS: Retrospective cohort study; RCT: Randomized controlled trial; RR: Risk ratio: SC: Small-cell: SRS: Stereotactic radiosurgery: UA: Unavailable; VMAT: Volumetric Mediated Arc Therapy; WBRT: Whole brain radio therapy

\section{Acknowledgements}

Not applicable.

\section{Funding}

IM received a scholarship from the KNAW to present the contents of this manuscript at the 2017 AANS meeting in Los Angeles.

\section{Availability of data and materials}

Not applicable.

\section{Authors' contributions}

$\mathrm{NL}$ screened the articles, conducted the review of literature and was responsible preparation of the manuscript. IM screened the articles, conducted the review of literature and was responsible preparation of the manuscript. LM screened the articles and conducted a review of literature. VB conducted the meta-analysis analysis. AD conducted a review of literature and helped to prepare the manuscript. HE was responsible of screening of the articles. BA was responsible of screening of the articles. SM was responsible of screening of the articles. JV was responsible for critical revision of the manuscript. CK was responsible for critical revision of the manuscript. TS was responsible for critical revision of the manuscript. RM conducted the metaanalysis was responsible for critical revision of the manuscript. MB was responsible for critical revision of the manuscript and provided senior input throughout the study. All authors read and approved the final manuscript.

\section{Competing interests}

The authors declare that they have no competing interests.

\section{Consent for publication}

Not applicable.

Ethics approval and consent to participate

Not applicable.

\section{Publisher's Note}

Springer Nature remains neutral with regard to jurisdictional claims in published maps and institutional affiliations.

\section{Author details}

${ }^{1}$ Cushing Neurosurgery Outcomes Center (CNOC), Department of Neurosurgery, Brigham and Women's Hospital, Harvard Medical School, Boston, MA, USA. ${ }^{2}$ Harvard Medical School, Boston, MA, USA. ${ }^{3}$ Department of Neurosurgery, Brain Center Rudolf Magnus, University Medical Center 
Utrecht, HP G03.124, PO Box 855003508 GA Utrecht, The Netherlands. ${ }^{4}$ School of Pharmacy, MCPHS University, Boston, MA, USA. ${ }^{5}$ Department of Radiation Oncology, University Medical Center Utrecht, Utrecht, The Netherlands.

\section{Received: 7 March 2017 Accepted: 13 June 2017}

\section{Published online: 24 June 2017}

\section{References}

1. Nayak L, Lee EQ, Wen PY. Epidemiology of brain metastases. Curr Oncol Rep. 2012;14:48-54.

2. Iorio-Morin C, Masson-Cote L, Ezahr Y, Blanchard J, Ebacher A, Mathieu D. Early Gamma Knife stereotactic radiosurgery to the tumor bed of resected brain metastasis for improved local control. J Neurosurg. 2014;121(Suppl):69-74.

3. Barnholtz-Sloan JS, Sloan AE, Davis FG, Vigneau FD, Lai P, Sawaya RE. Incidence proportions of brain metastases in patients diagnosed (1973 to 2001) in the Metropolitan Detroit Cancer Surveillance System. J Clin Oncol. 2004;22:2865-72.

4. Weil RJ, Mavinkurve GG, Chao ST, Vogelbaum MA, Suh JH, Kolar M, Toms SA. Intraoperative radiotherapy to treat newly diagnosed solitary brain metastasis: initial experience and long-term outcomes. J Neurosurg. 2015; 122:825-32.

5. Lee CH, Kim DG, Kim JW, Han JH, Kim YH, Park CK, Kim CY, Paek SH, Jung $\mathrm{HW}$. The role of surgical resection in the management of brain metastasis: a 17-year longitudinal study. Acta Neurochir. 2013;155:389-97.

6. Adult Central Nervous System Tumors Treatment (PDQ $\left.{ }^{\oplus}\right)-$ Health Professional Version [http://www.cancer.gov/types/brain/hp/adult-braintreatment-pdq-cit/section_6.1]. Accessed 5 Feb 2016.

7. Smith TR, Lall RR, Lall RR, Abecassis IJ, Arnaout OM, Marymont MH, Swanson KR, Chandler JP. Survival after surgery and stereotactic radiosurgery for patients with multiple intracranial metastases: results of a single-center retrospective study. J Neurosurg. 2014;121:839-45.

8. Mulvenna P, Nankivell M, Barton R, Faivre-Finn C, Wilson P, McColl E, Moore B, Brisbane I, Ardron D, Holt T, et al. Dexamethasone and supportive care with or without whole brain radiotherapy in treating patients with nonsmall cell lung cancer with brain metastases unsuitable for resection or stereotactic radiotherapy (QUARTZ): results from a phase 3, non-inferiority, randomised trial. Lancet. 2016;388:2004-14.

9. Schackert G, Steinmetz A, Meier U, Sobottka SB. Surgical management of single and multiple brain metastases: results of a retrospective study. Onkologie. 2001;24:246-55.

10. Deangelis LM, Mandell LR, Thaler HT, Kimmel DW, Galicich JH, Fuks Z, Posner JB. The role of postoperative radiotherapy after resection of single brain metastases. Neurosurgery. 1989;24:798-805.

11. Patchell RA, Tibbs PA, Regine WF, Dempsey RJ, Mohiuddin M, Kryscio RJ Markesbery WR, Foon KA, Young B. Postoperative radiotherapy in the treatment of single metastases to the brain: a randomized trial. JAMA. 1998; 280:1485-9.

12. Rades D, Raabe A, Bajrovic A, Alberti W. Treatment of solitary brain metastasis. Resection followed by whole brain radiation therapy (WBRT) and a radiation boost to the metastatic site. Strahlenther Onkol. 2004;180:144-7.

13. Kocher M, Soffietti R, Abacioglu U, Villa S, Fauchon F, Baumert BG, Fariselli L Tzuk-Shina T, Kortmann RD, Carrie C, et al. Adjuvant whole-brain radiotherapy versus observation after radiosurgery or surgical resection of one to three cerebral metastases: results of the EORTC 22952-26001 study. J Clin Oncol. 2011;29:134-41

14. Brennan C, Yang TJ, Hilden P, Zhang Z, Chan K, Yamada Y, Chan TA, Lymberis SC, Narayana A, Tabar $V$, et al. A phase 2 trial of stereotactic radiosurgery boost after surgical resection for brain metastases. Int J Radiat Oncol Biol Phys. 2014;88:130-6.

15. Chang EL, Wefel JS, Hess KR, Allen PK, Lang FF, Kornguth DG, Arbuckle RB, Swint JM, Shiu AS, Maor MH, Meyers CA. Neurocognition in patients with brain metastases treated with radiosurgery or radiosurgery plus whole-brain irradiation: a randomised controlled trial. Lancet Oncology. 2009;10:1037-44.

16. Moher D, Liberati A, Tetzlaff J, Altman DG, Group P. Preferred reporting items for systematic reviews and meta-analyses: the PRISMA statement. Plos Med. 2009;6:e1000097.

17. Schnell MC, Bova FJ, Larson DA, Leavitt DD, Podgorsak EB, Wu A: AAPM Report No. 54: Stereotacic Radiation therapy-Report of Task Group 42 Radiation Therapy Committee. [http://www.aapm.org/pubs/reports/rpt_54. PDF]. Accessed 1 Mar 2017.
18. Hsieh J, Elson P, Otvos B, Rose J, Loftus C, Rahmathulla G, Angelov L, Barnett GH, Weil RJ, Vogelbaum MA. Tumor progression in patients receiving adjuvant whole-brain radiotherapy vs localized radiotherapy after surgical resection of brain metastases. Neurosurgery. 2015;76:411-20.

19. Shaw E, Kline R, Gillin M, Souhami L, Hirschfeld A, Dinapoli R, Martin L. Radiation Therapy Oncology Group: radiosurgery quality assurance guidelines. Int J Radiat Oncol Biol Phys. 1993;27:1231-9.

20. Patel KR, Prabhu RS, Kandula S, Oliver DE, Kim S, Hadjipanayis C, Olson JJ, Oyesiku N, Curran WJ, Khan MK, et al. Intracranial control and radiographic changes with adjuvant radiation therapy for resected brain metastases: whole brain radiotherapy versus stereotactic radiosurgery alone. J Neurooncol. 2014;120:657-63.

21. The Newcastle-Ottawa Scale (NOS) for assessing the quality of nonrandomised studies in meta-analyses [http://www.medicine.mcgill.ca/ rtamblyn/Readings\%5CThe\%20Newcastle\%20-\%20Scale\%20for\%20 assessing\%20the\%20quality\%20of\%20nonrandomised\%20studies\%20in \%20metaanalyses.pdf].

22. Atkins D, Best D, Briss PA, Eccles M, Falck-Ytter Y, Flottorp S, Guyatt GH, Harbour RT, Haugh MC, Henry D, et al. Grading quality of evidence and strength of recommendations. BMJ. 2004;328:1490.

23. Statistical formulae for calculating some $95 \%$ confidence intervals. [http:// onlinelibrary.wiley.com/store/10.1002/9781444311723.oth2/asset/oth2. pdf? $v=1 \& \mathrm{t}=$ ix3ohhpi\&s=779d7d53e9dc26449df8e600badd96b6dc084d68]. Accessed 1 Dec 2016.

24. Elaimy AL, MacKay AR, Lamoreaux WT, Fairbanks RK, Demakas JJ, Cooke BS, Peressini BJ, Holbrook JT, Lee CM. Multimodality treatment of brain metastases: an institutional survival analysis of 275 patients. World J Surg Oncol. 201 1;9:69.

25. Gu XD, Cai YT, Zhou YM, Li ZY, Xiang JB, Chen ZY. Prognostic factors and multidisciplinary treatment modalities for brain metastases from colorectal cancer: analysis of 93 patients. BMC Cancer. 2015;15:902.

26. Hwang SW, Abozed MM, Hale A, Eisenberg RL, Dvorak T, Yao K, Pfannl R, Mignano J, Zhu JJ, Price LL, et al. Adjuvant Gamma Knife radiosurgery following surgical resection of brain metastases: a 9-year retrospective cohort study. J Neurooncol. 2010;98:77-82.

27. Salvati M, Cervoni L, Caruso R, Gagliardi FM. Solitary cerebral metastasis from melanoma: value of the 'en bloc' resection. Clin Neurol Neurosurg. 1996;98:12-4

28. Caroli M, Di Cristofori A, Lucarella F, Raneri FA, Portaluri F, Gaini SM. Surgical brain metastases: management and outcome related to prognostic indexes: a critical review of a ten-year series. ISRN Surg. 2011;2011:207103.

29. Soon YY, Tham IW, Lim KH, Koh WY, Lu JJ: Surgery or radiosurgery plus whole brain radiotherapy versus surgery or radiosurgery alone for brain metastases. Cochrane Database of Systematic Reviews. 2014;1(3). doi:10.1002/14651858.CD009454.pub2.

30. Andrews DW, Scott CB, Sperduto PW, Flanders AE, Gaspar LE, Schell MC, Werner-Wasik M, Demas W, Ryu J, Bahary JP, et al. Whole brain radiation therapy with or without stereotactic radiosurgery boost for patients with one to three brain metastases: phase III results of the RTOG 9508 randomised trial. Lancet. 2004;363:1665-72.

31. Kaidar-Person $O$, Zagar TM, Ewend M, Tzuk-Shina T, Marks LB. Frameless LINAC-based stereotactic radiation therapy to brain metastasis resection cavity without whole-brain radiation therapy: a systematic review. Pract Radiat Oncol. 2016;6(5):324-30.

32. Atalar B, Modlin LA, Choi CY, Adler JR, Gibbs IC, Chang SD, Harsh GR, Li G, Nagpal S, Hanlon A, Soltys SG. Risk of leptomeningeal disease in patients treated with stereotactic radiosurgery targeting the postoperative resection cavity for brain metastases. Int J Radiat Oncol Biol Phys. 2013;87:713-8.

33. Johnson MD, Avkshtol V, Baschnagel AM, Meyer K, Ye H, Grills IS, Chen PY, Maitz A, Olson RE, Pieper DR, Krauss DJ. Surgical Resection of Brain Metastases and the Risk of Leptomeningeal Recurrence in Patients Treated With Stereotactic Radiosurgery. Int J Radiat Oncol Biol Phys. 2016;94:537-43.

34. Ojerholm E, Lee JY, Thawani JP, Miller D, O'Rourke DM, Dorsey JF, Geiger GA, Nagda S, Kolker JD, Lustig RA, Alonso-Basanta M. Stereotactic radiosurgery to the resection bed for intracranial metastases and risk of leptomeningeal carcinomatosis. J Neurosurg. 2014;121(Suppl):75-83.

35. Trifiletti DM, Lee CC, Schlesinger D, Larner JM, Xu Z, Sheehan JP. Leukoencephalopathy After Stereotactic Radiosurgery for Brain Metastases. Int J Radiat Oncol Biol Phys. 2015;93:870-8.

36. Kotecha R, Damico N, Miller JA, Suh JH, Murphy ES, Reddy CA, Barnett GH, Vogelbaum MA, Angelov L, Mohammadi AM, Chao ST. Three or More Courses of Stereotactic Radiosurgery for Patients with Multiply Recurrent Brain Metastases. Neurosurgery. 2017;80(6):871-9. 
37. Fokas E, Henzel M, Hamm K, Surber G, Kleinert G, Engenhart-Cabillic R. Multidisciplinary treatment of brain metastases derived from colorectal cancer incorporating stereotactic radiosurgery: analysis of 78 patients. Clin Colorectal Cancer. 2011:10:121-5.

38. Matsuda R, Hironaka Y, Suigimoto T, Nakase H. Glioblastoma Multiforme in the Pineal Region with Leptomeningeal Dissemination and Lumbar Metastasis. J Korean Neurosurg Soc. 2015;58:479-82.

39. Mcpherson CM, Suki D, Feiz-Erfan I, Mahajan A, Chang E, Sawaya R, Lang FF. Adjuvant whole-brain radiation therapy after surgical resection of single brain metastases. Neuro Oncol. 2010;12:711-9.

40. Choi CY, Chang SD, Gibbs IC, Adler JR, Harsh GR, Lieberson RE, Soltys SG Stereotactic radiosurgery of the postoperative resection cavity for brain metastases: prospective evaluation of target margin on tumor control. Int J Radiat Oncol Biol Phys. 2012;84:336-42.

41. Lagerwaard FJ, Levendag PC, Nowak PJ, Eijkenboom WM, Hanssens PE, Schmitz PI. Identification of prognostic factors in patients with brain metastases: a review of 1292 patients. Int J Radiat Oncol Biol Phys. 1999;43: 795-803.

42. Sperduto PW, Kased N, Roberge D, Xu Z, Shanley R, Luo X, Sneed PK, Chao ST, Weil RJ, Suh J, et al. Summary report on the graded prognostic assessment: an accurate and facile diagnosis-specific tool to estimate survival for patients with brain metastases. J Clin Oncol, 2012;30:419-25.

43. Eaton BR, Gebhardt B, Prabhu R, Shu HK, Curran Jr WJ, Crocker I. Hypofractionated radiosurgery for intact or resected brain metastases: defining the optimal dose and fractionation. Radiat Oncol. 2013;8:135.

44. Lima LC, Sharim J, Levin-Epstein R, Tenn S, Teles AR, Kaprealian T, Pouratian N. Hypofractionated Stereotactic Radiosurgery and Radiotherapy to Large Resection Cavity of Metastatic Brain Tumors. World Neurosurg. 2017:97:571-9.

45. NCT01372774: Stereotactic Radiosurgery or Whole-Brain Radiation Therapy in Treating Patients With Brain Metastases That Have Been Removed By Surgery [https://clinicaltrials.gov/ct2/show/NCT01372774]. Accessed 5 Feb 2017.

\section{Submit your next manuscript to BioMed Central and we will help you at every step:}

- We accept pre-submission inquiries

- Our selector tool helps you to find the most relevant journal

- We provide round the clock customer support

- Convenient online submission

- Thorough peer review

- Inclusion in PubMed and all major indexing services

- Maximum visibility for your research

Submit your manuscript at www.biomedcentral.com/submit 